\section{Abdominal sacrocolpopexy with prophylactic Burch colposuspension}

A randomized trial published in The New England Journal of Medicine shows that performing Burch colposuspension at the time of abdominal sacrocolpopexy for pelvic-organ prolapse significantly reduces the risk of developing postoperative stress urinary incontinence.

In the Colpopexy and Urinary Reduction Efforts (CARE) trial, 322 women with prolapse, who had no preoperative symptoms of stress incontinence, were randomly allocated to undergo abdominal sacrocolpopexy either with $(n=157)$ or without $(n=165)$ Burch colposuspension. Surgeons were blinded to the results of preoperative urodynamic assessment.

The primary endpoint was urinary stress incontinence and urge symptoms 3 months after surgery. Enrollment was prematurely terminated when the US team conducting the study noted that almost twice as many women who underwent sacrocolpopexy alone reported stress incontinence symptoms, compared with those who underwent sacrocolpopexy with concomitant Burch colposuspension (44.1\% and $23.8 \%$, respectively; $P<0.001)$. The protective effect of Burch colposuspension occurred irrespective of the patients' preoperative urodynamic status. There were no significant differences between the two groups in the incidence of other postoperative lower urinary tract symptoms, including urge incontinence.

The authors acknowledge that follow-up was short and that their results are not applicable to other surgical procedures for prolapse or incontinence. Long-term follow-up of these patients will determine whether the improvement in urinary control afforded by a prophylactic Burch colposuspension at the time of abdominal colpopexy will stand the test of time.

Original article Brubaker L et al. (2006) Abdominal sacrocolpopexy with Burch colposuspension to reduce urinary stress incontinence. N Engl J Med 354: 1557-1566

\section{PSA decline is a possible surrogate endpoint for survival of prostate cancer}

At present, the FDA accepts only survival as an endpoint in phase III trials of drug efficacy. As a result, trials of new drugs can take years to show results. The identification of reliable surrogate endpoints could speed up trials, and, consequently, drug development and approval. Declines in serum PSA levels have previously been associated with improved prostatecancer survival, so members of the Southwest Oncology Group (SWOG) reviewed the PSA data of patients in the SWOG 99-16 trial as a potential marker of survival.

They found that declines in PSA level of at least $20-40 \%$ and PSA velocity at 3 months following initiation of treatment were possible surrogate endpoints for survival. The optimal surrogate, however, was a PSA-level decline of $\geq 30 \%$ in the first 3 months of treatment; the risk of death for these patients was reduced by more than half (hazard ratio $0.40,95 \% \mathrm{Cl}$ $0.32-0.50, P<0.001$ ).

Surprisingly, the often-suggested $\geq 50 \%$ decline in serum PSA just failed as a surrogate measure. The authors suggest that the small number of patients who showed $a \geq 50 \%$ decline might have reduced the statistical power of the analysis, and recommend that this endpoint should be investigated in future trials.

It should be remembered that serum PSA decline and PSA velocity might only be suitable surrogate endpoints in trials of prostate-cancer drugs that induce cell death. Drugs with different mechanisms will require separate analyses to identify possible surrogate endpoints. As is the case with all retrospective studies, these findings must be validated in independent, prospective trials.

Original article Petrylak DP et al. (2006) Evaluation of prostate-specific antigen declines for surrogacy in patients treated on SWOG 99-16. J Natl Cancer Inst 98: 516-521

\section{Immediate versus deferred androgen-deprivation therapy for localized prostate cancer}

European investigators have carried out a large, multicenter trial (EORTC 30891) comparing immediate and deferred androgen-deprivation therapy, in prostate cancer patients who refuse surgery or for whom potentially curative resection is contraindicated.

A total of 985 men with recently diagnosed, localized prostate cancer, but without detectable metastatic disease, were randomly allocated to receive either immediate $(n=493)$ or deferred $(n=492)$ androgen-deprivation therapy. 